gyrfalco geschossen habe. Das Exemplar befindet sich bei einem hiesigen Präparator und soll der wissenschaftlichen Prüfung zugänglich gemacht werden.

Herr B ünger spricht über ein interessantes Nest einer Schwanzmeise. Dasselbe stand im Garten des Herrn Haushofmeister Meyer in Klein-Glienicke dicht neben einer viel benutzten Laube im Taxus.

\title{
Schalow.
}

Matschie.

\section{Bericht über die Januar-Sitzung.}

Verhandelt Berlin, Montag, den 6. Januar 1896.

Anwesend die Herren: Grunack, von Treskow, Deditius, Krüger-Velthusen, Freese, Schulz, Schalow, Reichenow, Heck, G. Rörig, R. Rörig, Matschie, Schenkling, Schreiner, Günther u. Müller-Liebenwalde.

Von auswärtigen Mitgliedern: Herr A rend s (Juist).

Als Gast: Herr Dr. von Maehrenthal.

Vorsitzender: Herr Schalow. Schriftf.: Herr Matschie.

Herr Sch al ow eröffnet die Sitzung mit einem Vortrage über die Fortschritte aufdem Gebiete der Ornithologie während des Jahres 1895, soweit sie das palaearktische Gebiet betreffen.

Die Herren Reichenow und Matschie besprechen nun. mehr die im December 1895 erschienenen ornithologischen Arbeiten und Zeitschriften.

Herr Reichenow legt einige Neuerwerbungen des Königl. Museums für Naturkunde vor, ein Ei von Apteryx mantelli und je 1 Exemplar von Cyanorhamphus unicolor u. Nesonetta aucklandica.

Derselbe teilt alsdann mit, dass Cannabina linaria von Hn. v. T s ch usi bei Hallein in diesem Winter sehr spät beobachtet, hier bei Berlin von ihm selbst dagegen schon im November gesehen worden sei. Auch Schneeammern und Schneeeulen sind im December zahlreich beobachtet worden. Der hiesige Präparator B ock hat mehrere Schneeeulen zum Ausstopfen erhalten.

Herr S chalow bemerkt, dass am 15. December eine Schneeeule bei Swinemünde erlegt worden ist.

Schalow.

Matschie.

\section{Bericht über die Februar-Sitzung.}

Verhandelt Montag, den 3. Februar 1896.

Anwesend die Herren: Günther, Stoll, Deichler, Grunack, Thiele, von Treskow, Arends, Freese, 
Schenkling-Prevost, Schulz, von Erlanger, Schalow, Matschie, Reichenow, Bünger, Deditius, N auwerck, Pascal, R. Rörig, Walter, v. Oertzen u. Krüger-Velthusen.

Von auswärtigen Mitgliedern:Hr.Z i m m er m a n n(Königsberg). Als Gast: Herr Dr. Kra em er (Berlin).

Vorsitzender: Herr Schalow. Schriftf.: Herr Matschie.

Nach Vorlesung und Annahme des Berichtes über die Februar-Sitzung bespricht zunächst Herr Reichenow einige neu eingegangene ornithologische Schriften.

Herr Matschie legt hierauf die neuesten Nummern mehrerer Zeitschriften vor, in denen Mitteilungen über Vogelkunde enthalten sind. Über das Schwimmvermögen der Hühnervögel, namentlich von Puten und jungen Fasanen ist in mehreren Journalen berichtet worden. Der Referent teilt einen Fall mit, in dem die Schwimmfertigkeit eines Haushuhnes beobachtet worden war. Auf einem Gute bei Pyritz waren einer Henne Enteneier untergelegt worden. Nach dem Auskriechen begaben sich die jungen Vögelchen bald ins Wasser. Die Glucke stürzte sich ihren Pflegebefohlenen nach und trieb sie schwimmend von dem Teich herunter. Sie entwickelte eine solche Ausdauer in ihren Bewegungen auf dem ungewohnten Elemente und verstand es, so geschickt die kleinen Enten von dem Wasser zu verjagen, dass sie deswegen von ihrem Ehrenposten als Entenmutter entbunden werden musste.

Herr Schalow knüpft an ein von Herrn Reichenow über die Vögel von Aden gegebenes Referat die Bemerkung, dass Lanius lathora, der in der betreffenden Arbeit für den Südrand Arabiens aufgeführt war, auch in Nord-Afrika nach mehreren Autoren beobachtet sein soll. Die grauen Würger des Mittelmeer-Gebietes bedürfen noch sehr eingehender Bearbeitung.

Alsdann hält Herr Schulz einen längeren Vortrag über das Vogelleben am unteren Amazonas. Die Sammlungen des Reisenden werden im Museum ,von Berlepsch" aufbewahrt. Unter den Vogelarten, welche Herr Schulz nach Europa schickte, verdient die herrliche Pipra opalicans besondere Aufmerksamkeit.

Herr Stoll zeigt nunmehr Sperbereulen und weissrückige Spechte aus Livland vor, welche durch vorzügliche Präparation allgemeines Interesse erregen.

Herr Schenkling teilt aus einem französischen Journal eine Notiz über die Verbreitung der Tuberkulose durch einen 
Papagei mit. Die in dem Artikel behauptete acute Wirkung des Tuberkulose-Bacillus wird durch Herrn Dr. K r a e m er angezweifelt.

Herr Schalow spricht über den seiner Zeit bereits von Herrn vo n Dallw itz erwähnten, im Wohlauer Kreise bei Mönchmotschelwitz erlegten Jagdfalken. Das Exemplar, welches für das Kgl. Museum für Naturkunde angekauft worden ist, hat sich als ein Weibchen im Übergangskleide von Falco rusticulus L. herausgestellt. Der Vortragende hebt hervor, dass dieses Stück das zweite nachweislich in Deutschland erlegte Exemplar des norwegischen Jagdfalken darstellt. Das andere ist in der Nähe von Hamburg geschossen und von Hartert bestimmt worden. In England wurde vor circa 10 Jahren ein anderes Stück bei Suffolk erlegt. Bisher ist nur $F$. rusticolus für Deutschland nachgewiesen, alle Mitteilungen über das Vorkommen von $\boldsymbol{F}$. islandicus und $\boldsymbol{F}$. candicans innerhalb der Grenzen unseres Vaterlandes sind sehr unsicher.

Schalow.

Matschie.

\section{Bericht über die März-Sitzung.}

Verhandelt Berlin, Montag, den 2. März 1896. Abends 8 Uhr im Sitzungslokale, Bibliothekzimmer des Architekten-Vereinshauses, Wilhelmstr. 92. II.

Anwesend die Herren: Reichenow, Grunack, Thiele, Emcke, von Treskow, Deditius, Schenkling, Stoll, Günther, Schulz, Deichler, B rehm, Bünger, R. Rörig, G. Rörig, Krüger-Velthusen, Pascal, Matschieu. Arends.

Als Gast: Herr. Gotts chlag.

Vorsitzender: Herr Reichenow. Schriftf.: Herr Matschie.

Nachdem die Herren Reichenow und Matschie über die während des verflossenen Monats erschienenen und eingegangenen ornithologischen Arbeiten berichtet haben, spricht Herr G. Rörig über die Verbreitung der Zwergtrappe in Deutschland. Der Vortrag, welcher in No. 47 der „Deutschen Jägerzeitung“ abgedruckt ist, falst die in der Litteratur aufgezählten Mitteilungen über das Auftreten der Zwergtrappe in Deutschland zusammen.

Herr Reichenow bringt eine Petition des „Vereins zur Bekämpfung des Vogelmassenmordes" an den Reichstag zur Sprache und ist der Ansicht, dafs die Deutsche Ornithologische Gesellschaft Mafsregeln gegen die Verwendung von Vogelkörpern zu Modezwecken zustimmen könne, aber gegen weitere Be- 


\section{$2 \mathrm{BHL}$ Biodiversity Heritage Library}

1896. "Bericht über die Februar-Sitzung. Verhandelt Berlin, Montag, den 3. Februar 1896." Journal

$f u$

r Ornithologie 44, 251-253. https://doi.org/10.1007/bf02209523.

View This Item Online: https://www.biodiversitylibrary.org/item/101723

DOI: https://doi.org/10.1007/bf02209523

Permalink: https://www.biodiversitylibrary.org/partpdf/142313

\section{Holding Institution}

Smithsonian Libraries

\section{Sponsored by}

Biodiversity Heritage Library

\section{Copyright \& Reuse}

Copyright Status: Public domain. The BHL considers that this work is no longer under copyright protection.

This document was created from content at the Biodiversity Heritage Library, the world's largest open access digital library for biodiversity literature and archives. Visit BHL at https://www.biodiversitylibrary.org. 